\title{
Effect of Immediate Temporization on Pink Esthetic Score in Immediate Implant Placement in Anterior Maxilla Versus No Temporization - A Randomized Controlled Clinical Trial
}

\author{
Ahmed AbdAllah El Sayed Badr*, Ass. Prof.dr. Hani El-Nahass** \\ and Dr. Ibrahim El-Refai***
}

Effect of Immediate Temporization on Pink Esthetic Score in Immediate Implant Placement in Anterior Maxilla Versus No Temporization

A Randomized Controlled Clinical Trial

\section{Introduction}

A frequent fault in implant literature has been the failure to recognize the complex multidimensional problem associated with the insertion of a foreign device into the body and the expectation that it should remain preferably functional, over the life span of the patient. (Albrektsson et al. 1981). Despite that osseointegration of oral implants is a predictable consequence of surgical placement, a pre-operative assessment of the bone volume and quality may allow a successful outcome of implant placement (Jacobs \& van Steenberghe 1998). Lazzara (1989) was the first to study immediate implant placement in humans using thread-type implants with ePTFE membranes. Implants were placed $2 \mathrm{~mm}$ sub-crestal to allow bone remodeling and osseous regeneration up to the cover screw. The study concluded that immediate implant placement could allow bone preservation at the extraction sites. Implant placement in fresh extraction sockets, was reported to act as predictable and successful technique, affording fewer surgical interventions for 31 years, since first being introduced for the first time in 1976 by Schulte and Heimke as an alternative to the original Branemark two stage protocol.

* Master Degree Of Implantology

** Professor of Oral Medicine and Periodontology Cairo University

*** Lecturer of Oral Medicine and Periodontology Cairo University 


\section{Aim of the study}

The aim of the study is to clinically investigate the soft tissue profile and corresponding esthetic score over 1 year following immediate implant placement, in Immediate implant with immediate temporization versus no temporization .

\section{Subjects and methods}

\section{I) Materials:}

a- Chemicals

b-Patients Selection

\section{Inclusion criteria:}

- Patients who have at least one irrestorable tooth in the esthetic zone that needs to be extracted.

- Patients with healthy systemic condition (Medically free).

- Patients aged from 18 to 65 years old.

Buccal bone thickness should be at least $1 \mathrm{~mm}$. verified by CBCT

- Sinus floor or nasal floor clearance from root apex at least $4 \mathrm{~mm}$.

- Good oral hygiene.

- Accepts one year follow-up period (cooperative patients).

- Patient provides an informed consent.

\section{Exclusion criteria}

a) Patients with parafunctional habits that produce overload on the implant (Lobbezoo et al. 2006).

b) Pregnant females (Wiener and WienerPla, 2014).

c) Smoking patients (Lambert et al. 2000).

After initial screening, a total of 19 patients (males and females) were recruited in the present study, with ages ranged from 21-45 years and having at least one missing tooth in the esthetic zone need

\section{Allocation: (Random sequence generation):}

Methods of generating randomization:

Patients will be randomly selected using computer generated randomization (www. randomizer.org) and will be performed by another individual other than the investigator. The numbers will be allocated to each patient.

The patients were randomly allocated into two groups:

- Prospective study, parallel groups, Randomized Controlled Clinical Trial. Allocation ratio 1:1

- Patients are to be selected from the outpatientclinic of the department of Oral Medicine and PeriodontologyCairo University.

c- The patients were randomly allocated into two groups

\section{Methods:}

\section{Study Design}

1-Preoperative procedure:

2-Initial therapy:

3-Clinical examination:

4- Radiographic examination

5-General Surgical procedure

6- Postoperative Care

\section{Patient self-care instructions:}

1) Application of an ice bag to the treated area for the first 24 hours as previously recommended by (Francesco et al., 2012).

2) The patients were instructed to gently brush the operated area starting from the second day with a soft brush using roll technique. 
Pink Esthetic Score assesses 7 soft tissue variables, it allows for reproducible assessments of peri-implant soft tissue around single tooth implants

The contralateral tooth and the adjacent soft tissue served as reference. Using a 0-12 scoring system, with 0 being the lowest and 2 being the highest value, the maximum achievable PES was 14.

At the second assessment, the photographs were scored in reverse order.

\section{Statistical analysis:}

Data management and statistical analysis were performed using the Statistical Package for Social Sciences (SPSS) version. 24. Numerical data were summarized using means and standard deviations or medians and ranges.

Data were explored for normality by checking the data distribution and using Kolmogorov-Smirnov and Shapiro-Wilk tests.

Categorical data (gender) were summarized as percentages. For categorical variables, differences were analyzed with chi square (2) test and Fisher's exact test when appropriate.

For PES summation score comparisons between 2 groups and overtime was done by mixed ANOVA with repeated measure. For more details comparisons between the 2 groups regarding summation of PES score were done using the t-test and overtime in each group was done by repeated measure ANOVA followed by paired t-test for pairwise comparisons. Adjustments of $p$ value were done using the Bonferroni method for multiple testing. All $p$-values are two-sided. The significance of the differences among treatment groups, to all statements of significance were based on the probability of $\leq 0.05$

\section{Results}

\section{A. Demographic Data:}

Data for gender are presented in

19 patients with 22 implants with irrestorabile tooth in the esthetic upper region indicated for extraction \& immediate implant placement participated in this study $(7$ males, 12 females). They were randomly divided into two equal groups:

Group A: Immediate implant placement with temporization

Group B: Immediate implant placement with no temporization

\section{Gender distribution in both groups:}

Gender distribution in Group (A) involved 4 males and 5 females while in Group (B) involved 3 males and 8 females. There was no significant difference between both groups for gender $(\mathbf{P}=\mathbf{0 . 6 4 2})$.

\section{B) Post-Surgical Patient Satisfaction Questionnaire (PSPSQ)}

\section{B) PES:}

1- Mesial papilla:

2- Distal papilla:

3-Curvature of facial mucosa:

4- Soft tissue color:

5- Soft tissue texture:

6-Gingival margin:

\section{Total PES score:}

After 3 months, the mean PES for group A was $9.82 \pm 1.08$ and was $8.45 \pm 1.86$ for group B.

After 6 months, the mean PES for group A was $9.91 \pm 1.38$ and was $9.5 \pm 1.72$ for group $B$.

After 9 months, the mean PES for group A was $10.36 \pm 1.03$ and was $9.27 \pm 1.56$ for group $\mathrm{B}$.

Overall, there was no difference between groups $(\mathbf{P}=\mathbf{0 . 1 7 0})$, the time effect was statistically significant $(\mathbf{P}=\mathbf{0 . 0 1 4})$ and the interaction term was statistically not significant

Total PES score comparisons between groups:

There was no difference between groups at different time points, all $\mathrm{P}$ values $>0.05$ 
Total PES score comparisons overtime in each group:

There was no difference between different time points in group $\mathrm{A}$, while there was statistically significant difference in group $B$ $\mathbf{P}=\mathbf{0 . 0 0 1}$. Pairwise comparisons revealed that after 3 months is statistically significant from 6 months and 9 months as well.

\section{(C) Gingival Recession:}

\section{(E) BUCCAL BONE:}

bone comparisons between groups: Buccal

There was no difference between groups at different time points, all $P$ values $>0.05$

Buccal bone comparisons overtime in each group:

There was no difference between different time points in group B, while there was statistically significant difference in group $A$ $\mathbf{P}<\mathbf{0 . 0 0 1}$. Pairwise comparisons revealed that all times are statistically significant from each other's

\section{(f) Mucosal biotype:}

Mucosal biotype comparisons between groups:

There was no difference between groups at different time points, all $P$ values $>0.05$

Mucosal biotype comparisons overtime in each group:

There was statically significant difference between different time points in both groups. Pairwise comparisons revealed that at 3 months was statically significant from 9 months Case indix
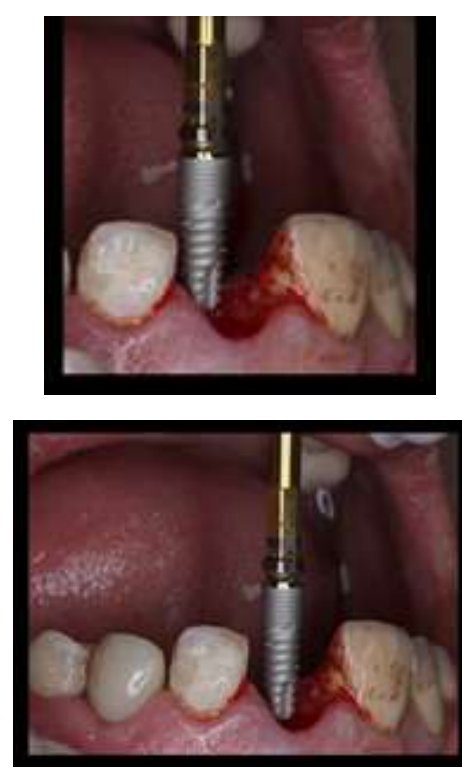

Atraumatic extraction of upper Central
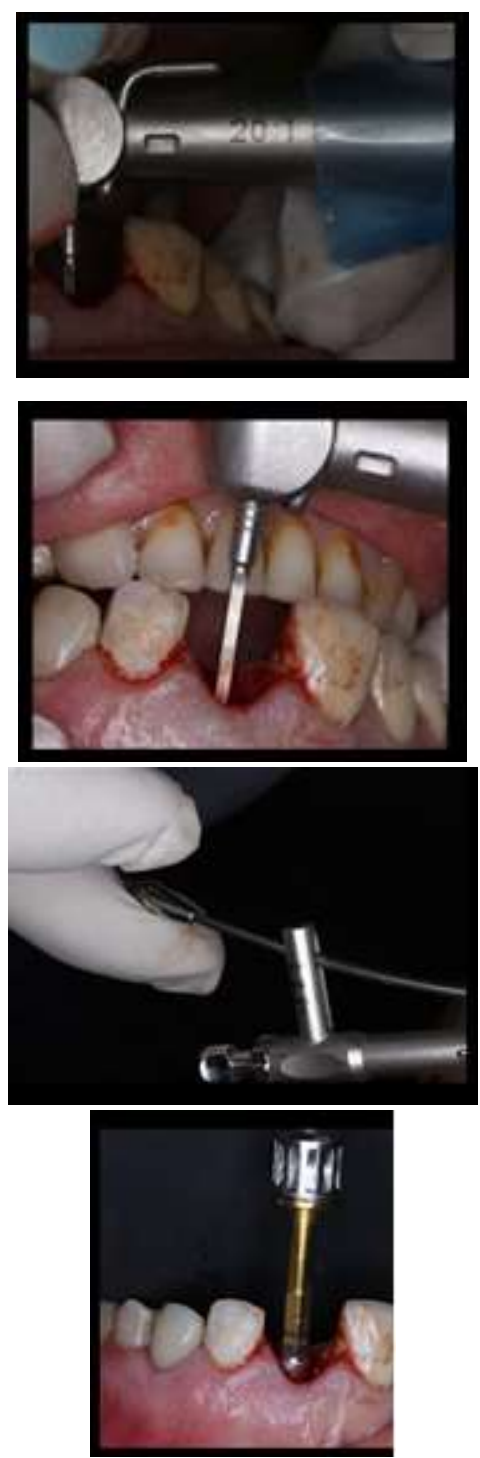


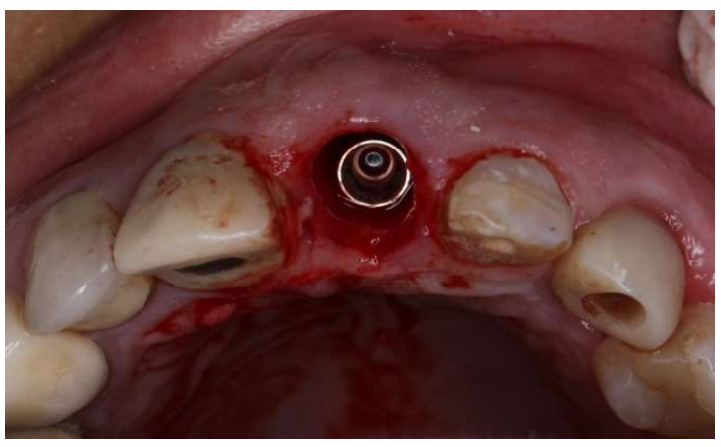

Check implant stability after insertion of implant body
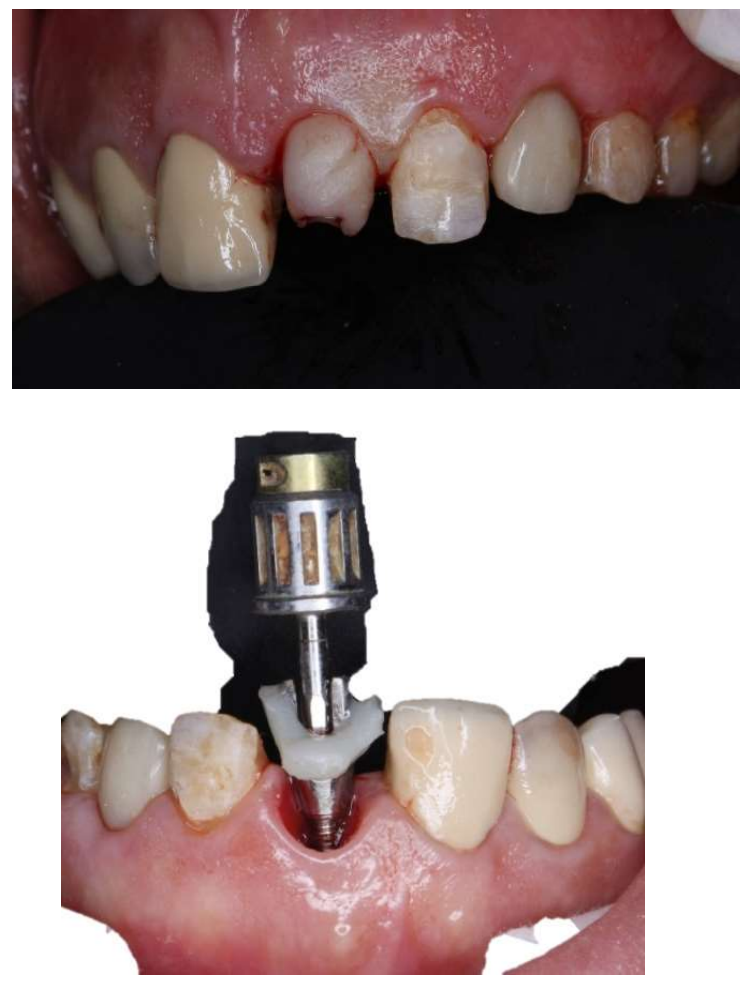

Screw retained temporary restoration inserted into implant body

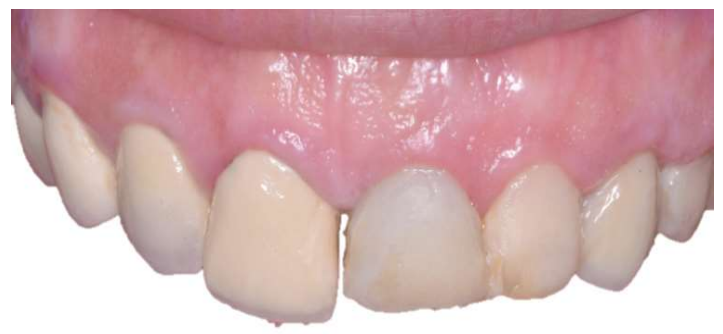

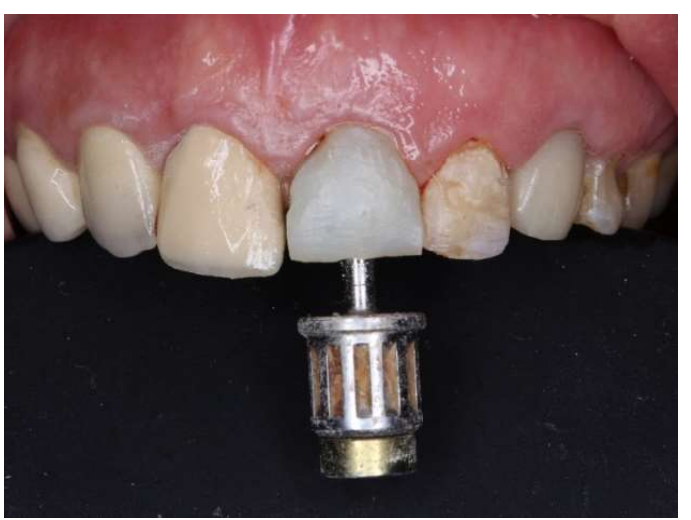

Temporization
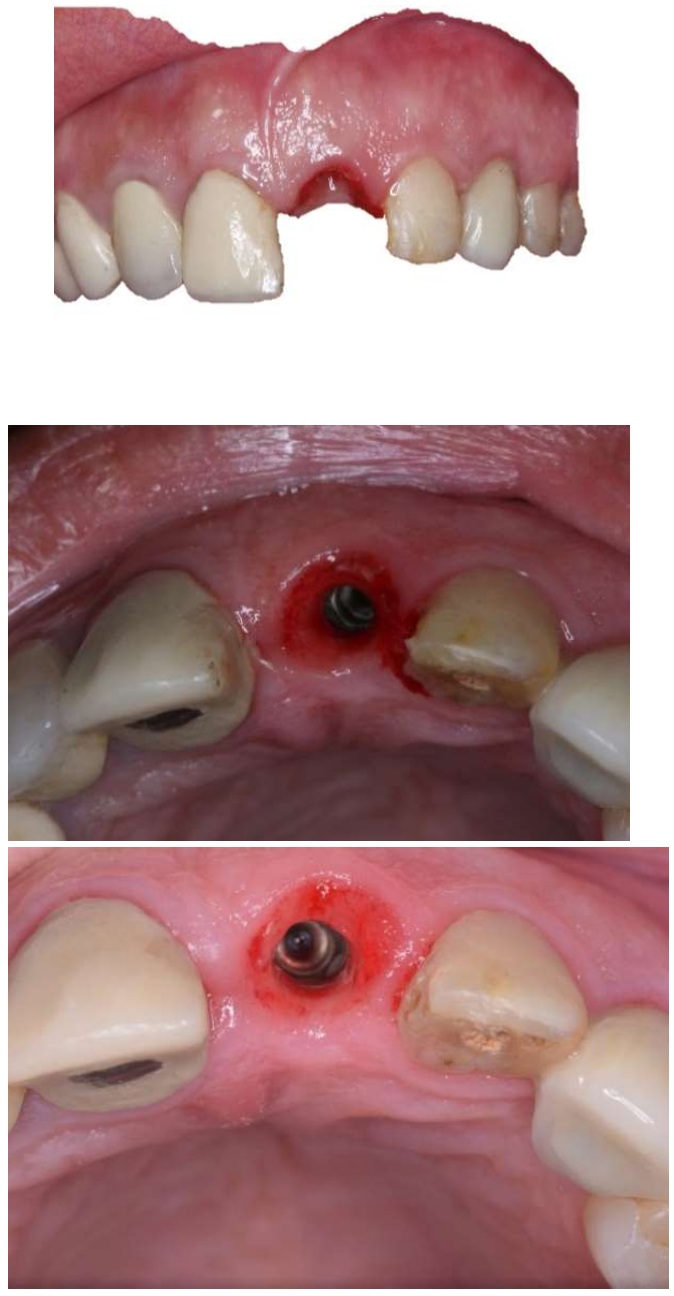


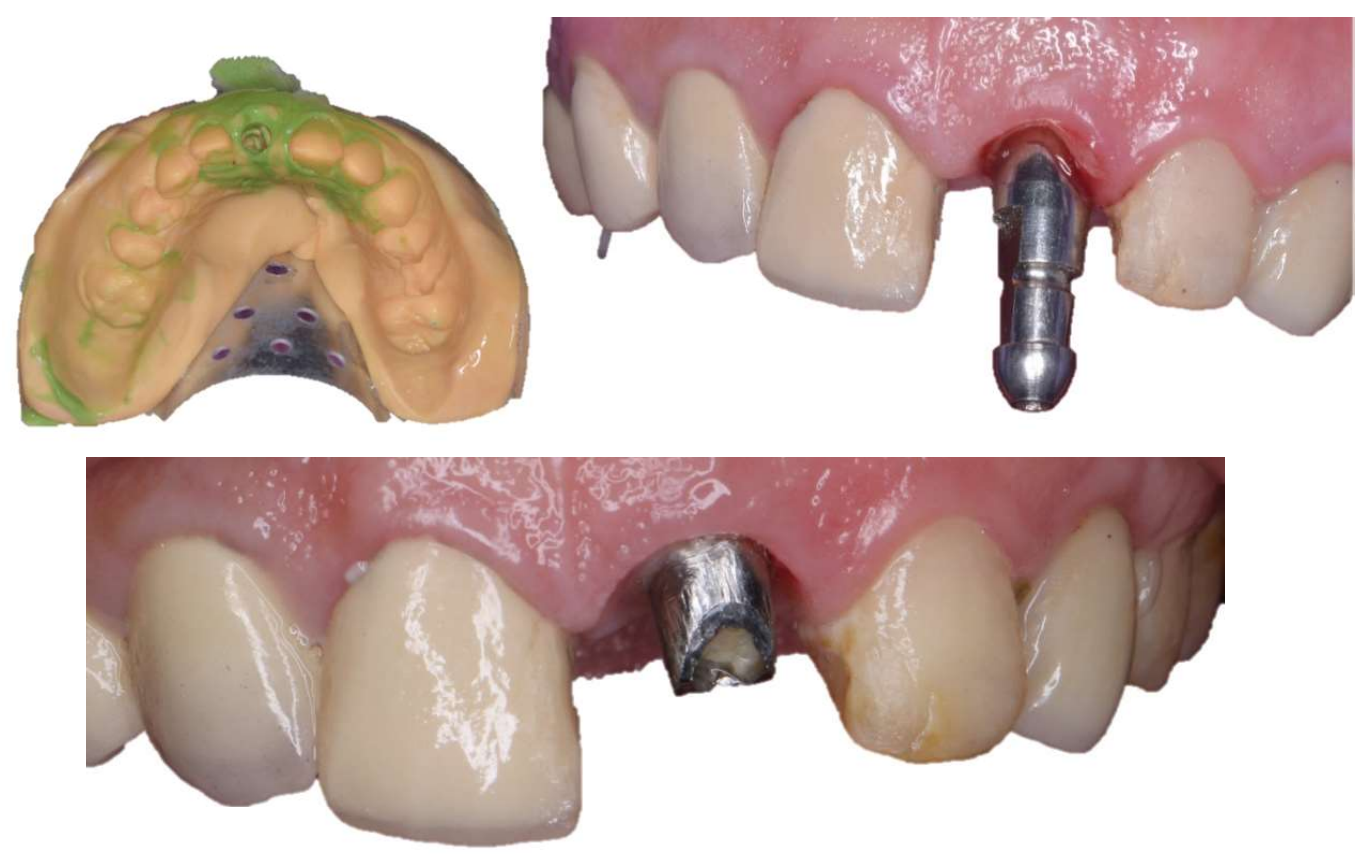

Emergence profile before 2nd impression 2nd impressio
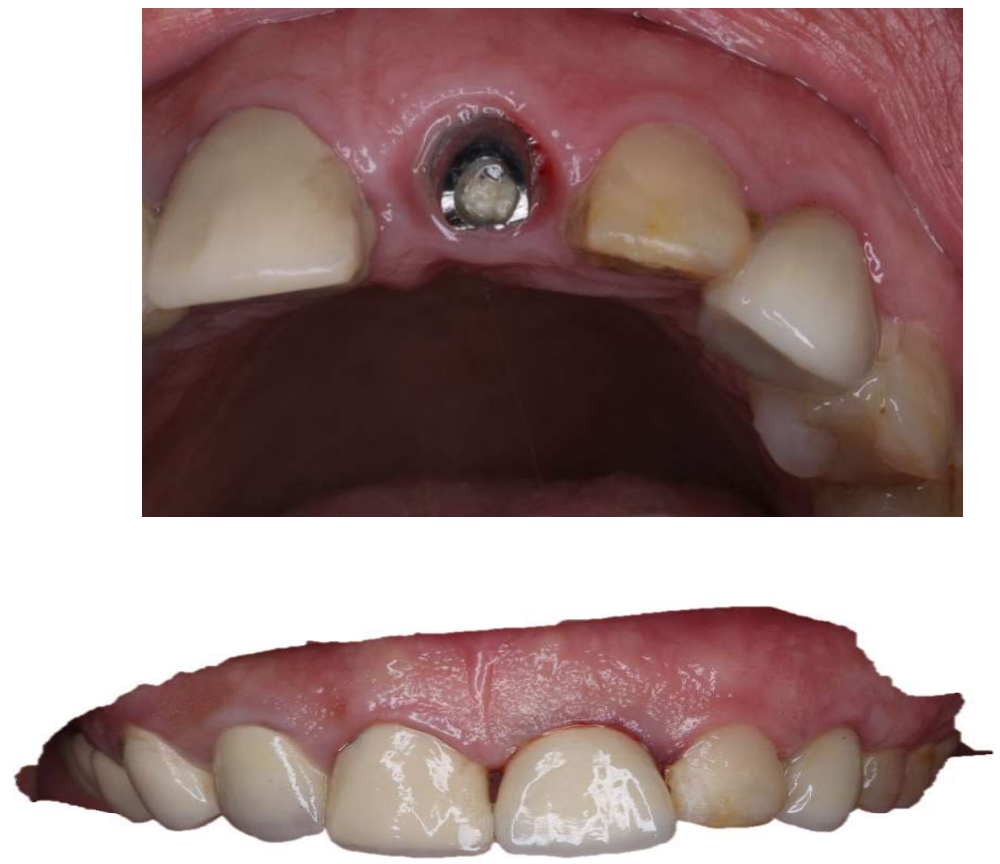


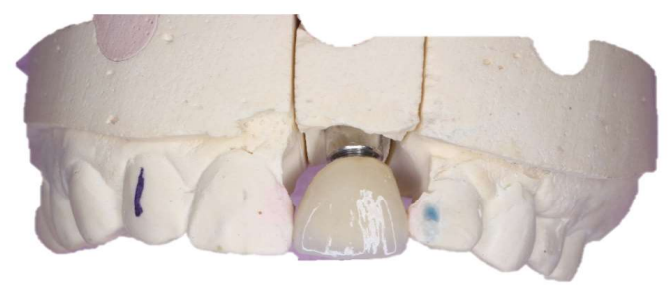

n Final crown

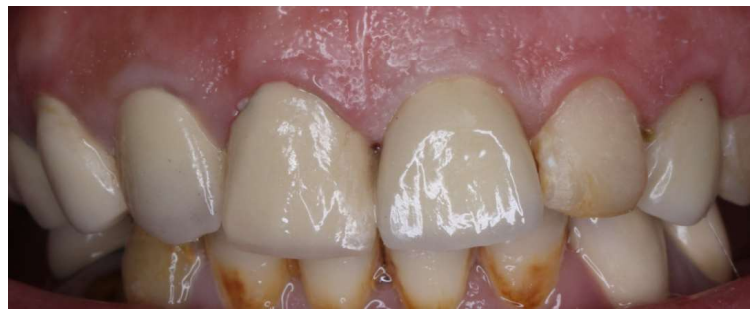

Final crown (Follow up after 8 months)

\section{Discussion:}

This study compared the corresponding esthetic score, gingival recession, patient morbidity and satisfaction following immediate implant placement with temporization to that without temporization in 20 patients (22 implants), randomly enrolled from the outpatient clinic in the Faculty of Oral and Dental Medicine, Cairo University. Pink esthetic score (PES) is an evaluation system commonly used to assess esthetic clinical outcomes of soft tissue following implant placement (Lee et al., 2015), (Belser et al. 2009). The PES is claimed to be a reproducible objective score valid to assess the esthetics of implant supported restoration and adjacent soft tissue (Cho et al. 2010). To our best knowledge, this study is the first to assess the effect of temporization in PES and gingival thickness. Exclusion criteria comprised: 1) active smoking as recommended by Lambert and his colleagues who observed that the increased implant failures in smokers are due to exposure of peri-implant tissues to tobacco constitutes (Lambert et al. 2000), 2) history of para-functional habits including clenching and bruxism to avoid an overload on the implants, accelerated bone loss and consequently implant failure (Lobbezoo et al.
2006). 3) ongoing pregnancy as pregnancy $\square$ s hormonal shifts alter the gingival tissue's immunological response at the interdental papillae (Wiener and Wiener-Pla 2014). Moreover, many interventional protocols are necessary during the trial such as the use of $\mathrm{x}$-rays, anesthesia and antibiotics which are all contraindicated during pregnancy)

\section{Summary:}

The aim of the study is to clinically investigate the soft tissue profile and corresponding esthetic score over 1 year following immediate implant placement, in Immediate implant with immediate temporization versus no temporization. 22 implants were placed in 19 patients; 11 implants for each group and measure the change in PES, soft tissue change ever 3,6 and 9 months. The control group which included 10 patients with no temporization after immediate implant placement, while the study group which included 9 patients with temporization at the time of implant placement. The temporary abutment was fabricated and fixed at the time of surgery also the final restorations were fabricated and fixed at the 3rd month postoperatively and the pink esthetic score was assessed. The results of the present study showed that, there was no statistically significant difference in PES between the two groups at the baseline and throughout the follow-up period. In the current study, there was a significant increase in the tissue thickness at $1 \mathrm{~mm}$ level in both groups at the end of the follow up period. However, at 2 $\mathrm{mm}$ level at 9 months there was a statistically significant increase with temporization (study) group only. The current study did not show any statistically significant difference between the two groups in the pink esthetic score. Thus, it could be reasoned that the temporization is a valid technique in increasing the emergence profile without any clinically significant enhancement in esthetics when compared to immediate implant placement without any temporization. 


\section{Conclusion:}

The temporization is an effective method to increase emergence profile and soft tissue around dental implants. The temporization and immediate implants placement without any bone grafting result in very good esthetic and bone thickness after 9 months follow up. The Temp pins and temporization technique allow for the simple and efficient postsurgical temporization of dental implants. We recommend that temporization should be used at the time of surgery which have a great effect on the emergence profile producing good result at the 2 nd stage for fabrication of final restoration. We recommend at the time of 2 nd stage to add flowable composite or Duralay attached to transfer copping to transfer the shape of emergence profile to lab technician.

\section{References:}

1) Abraham, S. et al., 2014. Gingival biotype and its clinical significance - A review. The Saudi Journal for Dental, pp.3—7.

2) Adell, R. et al., 1981. A 15-year study of osseointegtutcd implants in the treatment of the edentulous jaw. International journal of oral surgery, I pp.387-416.

3) Albrektsson T, Jacobsson M. Bone-metal interface in osseointegration. J Prosthet Dent. 1987; 57:5-10.

4) Albrektsson, T, G Zarb, P Worthington, and A R Eriksson. 1986. -The Long-Term Efficacy of Currently Used Dental Implants: A Review and Proposed Criteria of Success. The International Journal of Oral \& Maxillofacial Implants 1 (1): 11-25.

5) Albrektsson, T., Brånemark, P.-I., Hansson, H.-A., and Lindström, J. Osseointegrated titanium implants. Requirements for ensuring a long-lasting direct bone-to-implant anchorage in man. Acta Orthop Scand 52:155-170, 1981.

6) Alla RK, Ginjupalli K, Upadhya N, Shammas M, Rama Krishna R, Ravichandra S. Surface roughness of implants: A review. Trends Biomat Artif Org. 2011; 25(3):112.
7) American Dental Association, Council on Dental Materials, Instruments and Equipment. Provisionally acceptable endosseous implant for use in selected cases, Wozniak, W.T., in litt, 1985.

8) Araujo MG, Lindhe J. Dimensional ridge alterations following tooth extraction. An experimental study in the dog. J Clin Periodontol 2005; 32:212-218.

9) Araujo MG, Sukekava F, Wennstro “m JL, Lindhe J. Tissue modeling following implant placement in fresh extraction sockets. Clin Oral Implants Res 2006;17: 615-624.

10) Araújo, M.G. \&Lindhe, J., 2005. Dimensional ridge alterations following tooth extraction. An experimental study in the dog. Journal of clinical periodontology, 32(2), pp.212-8.

11) Artzi, $Z$ et al., 1993. Mucosal considerations for osseointegrated implants. The Journal of prosthetic dentistry, 70(5), pp. $427-32$.

12) Avila G, Galindo P, Rios H, Wang HL.Immediate implant loading: current status from available literature. Implant Dent. 2007; 16:235- 245.

13) Batal, H., Yavari, A. \& Mehra, P., 2015. Soft Tissue Surgery for Implants. Dental clinics of North America,59(2), pp.471—491.

14) Belser UC, Grutter L, Vailati F, Bornstein MM, Weber HP, Buser D. Outcome evaluation of early placed maxillary anterior singletooth implants using objective esthetic criteria: a cross-sectional, retrospective study in 45 patients with a 2- to 4-year follow-up using pink and white esthetic scores. J Periodontal 2009; 80:140-h Body Mass Index Percentile in Giza Governorate: Cross sectional study

Belal Y. Mahmoud*, Hala M. Abdel Majed $^{* *}$, EmanM. Eldin*** ${ }^{* *}$ FadyH. Elsayed ${ }^{* * * *}$

\footnotetext{
* Master Degree in Orthodontics

** Professor of OrthodonticsFaculty of DentistryCairo University

*** Professor of OrthodonticsFaculty of DentistryCairo University

**** Professor of OrthodonticsFaculty of

DentistryCairo University
} 


\section{Introduction:}

The aim of this study was Assessment of Skeletal Maturation in Egyptian School Girls Using Middle Phalanx of the Middle Finger MP3 and its Relationship with Body Mass Index Percentile in Giza Governorate.

Methods: The study included 1403 adolescent females (ages ranged from 8 to 14 years). The sample was divided into 6 groups according to their age. Middle phalanx of the middle finger (MP3) of each subject was radiographed and the developmental stages were evaluated by the method of Rajogobal and Kansal.

Results: A statistically significant difference was found between mean age values at different MP3 growth stages. Pair-wise comparisons showed that the mean age at Stage $(F)$ was 9.4 years while Stage (FG) was 10.3 years. At Stage $(\mathrm{G})$ the mean value of the pubertal spurt age was $11.2 \pm 0.98$ years while the mean age for Stage $(\mathrm{H})$ was12.1 years, Stage $(\mathrm{HI})$ 12.6 years and Stage (I) 13 years of age. There was a statistically significant direct correlation between MP3 stages and BMI percentiles in each age group. An increase in MP3 stages is associated with an increase in BMI percentile and vice versa. 
